\title{
Multiobjective Real-Coded Genetic Algorithm for Economic/Environmental Dispatch Problem
}

\author{
Ragab A. El-SEHIEMY ${ }^{1}$, Mostafa Abdelkhalik El-HOSSEINI ${ }^{2}$, Aboul Ella HASSANIEN ${ }^{3}$ \\ ${ }^{1}$ Electrical Engineering Department, Faculty of Engineering-Kafrelsheikh University \\ elsehiemy@eng.kfs.edu.eg \\ ${ }^{2}$ Computers and Systems Engineering Department, Faculty of Engineering- Mansoura University \\ melhosseini@eng.mans.edu.eg \\ ${ }^{3}$ Faculty of Computers \& Information, Cairo University \\ aboitcairo@fci-cu.edu.eg
}

\begin{abstract}
This paper outlines the optimization problem of nonlinear constrained multi-objective economic/environmental dispatch (EED) problems of thermal generators in power systems and presents novel improved real-coded genetic optimization (MO-RCGA) algorithm for solving EED problems. The considered problem minimizes environmental emission and non-smooth fuel cost simultaneously while fulfilling the system operating constraints. The proposed MORCGA technique evolves a multi-objective version of GA by proposing redefinition of global best and local best individuals in multi-objective optimization domain. The performance of the proposed MO-RCGA enhanced with biased Initialization, dynamic parameter setting, and elitism is carried out. The validity and effectiveness of the proposed MORCGA is verified by means of several optimization runs accomplished at different population sizes on standard IEEE 30bus test system. Simulation results demonstrated the capabilities of the proposed MO-RCGA algorithm to obtain feasible set of effective well-distributed solutions.
\end{abstract}

Keywords: Multiobjective real-coded genetic algorithm, economic environmental dispatch (EED), security.

\section{Introduction}

The problem of Economic environmental dispatch (EED) is an important optimization task in fossil fuel fired power plant operation for allocating generation among the committed units. It aims at optimizing two conflicted objectives of fuel cost and emission level, simultaneously while satisfying all operational system constraints [1-3].

The EED problem is a large-scale highly nonlinear constrained optimization problem characterized by complex and nonlinear with heavy equality and inequality constraints characteristics [4].

Traditionally, electric power systems aim at operating in such a way that the total fuel cost is minimized regardless of the emission produced in the system. An increased public awareness regarding the harmful effects of atmospheric pollutants on the environment has been noticed with concentrating on the importance of environmental protection and the passage of the Clean Air Act Amendments of 1990 has forced the utilities to adapt their design and operational strategies in order to reduce pollution and atmospheric emissions of the thermal power plants [5].

Many algorithms are developed to alleviate the effects of emission as installation of pollutant cleaning equipment, switching to low emission fuels, replacement of the aged fuel-burners with cleaner ones, and emission dispatching. The fourth option is the recent interested costless option compared to the first three options. That option is not any installing or modifying the exited pollution equipment. Then, the problem that has attracted much attention is pollution minimization due to the pressing public demand for clean air [4-6].

As the concern of environmental pollution has been increased in recent decades as well as the dramatic growing of fuel costs assure the continuous necessity of improvement of optimization methodologies for efficiently solving EED problems.

Classical methods such as the lambda iteration method and gradient method have been applied to solve the EED problems. But unfortunately, these methods are not feasible in practical power systems owing to the non-linear characteristics of the generators and nonsmooth cost functions. Consequently, many powerful mathematical optimization techniques that are fast and reliable, such as non-linear programming and dynamic programming have been employed to solve the EED problems. But due to the non-differential and non-convex characteristics of the cost functions, these methods are also unable to locate the global optima [1, 3]. 
In recent years, modern search-based optimization techniques were developed as efficient alternative practical tools for nonlinear optimization problems. A wide range of improved techniques is used to tackle both EED objectives simultaneously as competing objectives. The salient search based methods are [4-8], niched Pareto genetic algorithm [4], differential evolution [6] fuzzy model with adaptive genetic algorithm [7] and Real-Coded Genetic Algorithm [8].

Fuzzy sets can also be applied for decision making in multiple objectives including various constraints, therefore, an interactive fuzzy satisfying method is suggested to solve ED/EED problems [7, 9-10], particle swarm optimization technique [11-13], Biogeographybased algorithm for solving different economic load dispatch problems [14-15], differential evolution assisted by interior point algorithm [16] ant colony optimization (ACO) [17], seeker optimization algorithm [18] firefly algorithm [19], non-dominated sorting genetic algorithm (NSGA) [20], niched Pareto genetic algorithm (NPGA) [20], strength Pareto evolutionary algorithm (SPEA) [20] and multiobjective fuzzy based on particle swarm optimization algorithm [11], multiobjective bacteria foraging [21], Modified Shuffled Frog Leaping Algorithm (MSFLA) [22], and fuzzy ranking based real coded genetic algorithm (FR-RCGA) [42].

The binary GA solves many optimization problems that stump traditional techniques. When the variables are naturally quantized, the binary GA fits nicely. However, when the variables are continuous it is more logical to represent them by floating-point numbers [23-27].

A novel real-coded GA will be developed that has a lot of features that makes it improved algorithm; rarely stuck in local optima. These features include Biased Initialization, Elitism, and Dynamic parameter setting. It has been widely confirmed that real-number encoding performs better than binary or Gray encoding for function optimizations and constrained optimizations for many reasons include:

- Binary encoding for function optimization problems is known to have severe drawbacks due to the existence of Hamming cliffs, pair of encodings having a large Hamming distance while belonging to points of minimal distance in phenotype space. For example, the pair 0111111111 and 10000000000 belongs to neighboring points in phenotype space (points of minimal Euclidean distance) but have maximum Hamming distance in genotype space [24]

- As the topological structure of the genotype space for real-number encoding is identical to that of the phenotype space, it is easy to form effective genetic operators by borrowing useful techniques from conventional ones [23].

- Also, the binary GA has its precision limited by the binary representation of variables; using floating point numbers instead easily allows representation to the machine precision [25].

- Real-coded GA also has the advantage of requiring less storage than the binary GA because a single floating-point number represents the variable instead of Nbits integers [23, 26].

This paper proposes Multiobjective real coded genetic algorithm (MO-RCGA) for simultaneously optimizing both economic and environmental objectives while achieving the operating system constraints. This EED problem is formulated as a nonlinear constrained optimization problem. In order to show the effectiveness of the proposed approach, problem solving is applied on standard IEEE 30 bus system.

\section{Problem Formulation}

The nonlinear optimization problem can be expressed in the form of a constrained optimization problem as:

Min $f(x)$

Subject to:

$\left\{\begin{array}{l}g(x)=0 \\ h(x) \leq 0\end{array}\right.$

where, $f(x)$ is the objective function such as generators fuel costs, transmission line losses, etc, $g(x)$ represents the equality constraints, $h(x)$ represents the inequality constraints, and $x$ is the vector of the controlled variables that may be generator real power outputs, generator voltages, switchable reactive power, and transformer tap setting. 
The combined economic-environmental dispatch issue is multidimensional, non-linear, non-convex and highly constrained problem. It involves multiple and often conflicting optimization criteria for which no unique optimal solution can be determined with respect to all criteria [5].

The economic-environmental power dispatch problem can be stated as finding the thermal unit generation and corresponding water release from each reservoir throughout all planning time intervals, so as to minimize the total cost of fuel and pollutants emission while satisfying load balance, thermal, and hydraulic constraints. The objectives and constraints considered in the formulation of the combined economic emission power dispatch problem are addressed in this section. The derivation of the optimal solution is based on the weighted- sum method of the two objective functions is considered.

In this paper, the objective functions are

The first one is the non-linear fuel cost of generators. The generators cost curves are represented by quadratic functions with sine components. The superimposed sine components represent the rippling effects produced by the steam admission valve openings. The total $\$ / h$ fuel cost can be expressed as:

$$
\begin{aligned}
\min F_{t} & =\sum_{i=1}^{N G} f_{i}\left(P G_{i}\right) \\
& =\sum_{i=1}^{N G} a_{i}+b_{i} P G_{i}+c_{i} P G_{i}^{2}+\mid d_{i} \sin \left[e_{i}\left(P G_{i}^{\text {min }}-P G_{i}\right)\right]
\end{aligned}
$$

Where,

- $F_{i}$ : is the non-linear objective function defining the total power generation cost of the system.

- $a_{i}, b_{i}, c_{i}, \mathrm{~d}_{\mathrm{i}}$ and $\mathrm{e}_{\mathrm{i}}$ are the coefficients of power generation cost function.

- NG: is the number of generation buses, and min. denotes to minimum value of the objective function.

The second objective aims at minimizing the emission effects. The atmospheric pollutants such as sulpher oxides and nitrogen oxides caused by fossil fueled thermal units can be modeled separately. However, for comparison purposes, the total ton/h emission of these pollutants can be expressed as [2-4] as:

$$
\begin{aligned}
\operatorname{minEmission}_{t} & =\sum_{i=1}^{N G} g_{i}\left(P G_{i}\right) \\
= & \sum_{i=1}^{N G} 10^{-2}\left(\alpha_{i}+\beta_{i} P G_{i}+\gamma_{i} P G_{i}^{2}\right)+\mid \xi_{i} \exp \left[\lambda_{i} P G_{i}\right]
\end{aligned}
$$

Where, $\alpha_{i}, \beta_{i}, \gamma_{i}, \xi_{\mathrm{i}}$ and $\lambda_{\mathrm{i}}$ are the coefficients of power generation cost function.

The objective functions in (3) and (4) are subjected to the following constraints:

\subsection{Equality constraints}

Two types of equality constraints are considered: one is to simulate the total power balance for the network and the other for representing the power balance at each bus.

\section{Active / reactive power balance constraints}

The generators real and reactive power outputs should be equal to the total load demand and transmission line losses, this constraint can be expressed as:

$$
\sum_{i=1}^{N G} P G_{i}=\sum_{j=1}^{N L} P D_{j}+P_{L}
$$

Where, $P G_{i}$ is the power generation at bus $i$, $P D_{j}$ is the load demand at load bus $j, N L$ is the number of load buses and $P_{L}$ is the total power losses in the system.

The transmission active power losses equal to the sum of the injected powers at all system buses. The active power injected can be calculated from Equ. (6) as:

$$
\begin{aligned}
& P_{i}=V_{i} \sum_{j \neq i} V_{j}\left(G_{i j} \sin \theta_{i j}+B_{i j} \sin \theta_{i j}\right) \\
& P_{L}=\sum\left(P_{i}\right)
\end{aligned}
$$

$i \in \mathrm{n}$, where, $i$ is set of numbers of buses except swing Bus, $V_{i}$ is voltage magnitude of bus $i, \theta_{i}$ is voltage phase angle of bus $i, \theta_{i j}$ is phase angle difference between buses $i$ and $j$,

$\mathrm{G}_{i j}$ is mutual conductance between buses $i$ and $j$, and $B_{i j}$ is mutual susceptance between buses $i$ and $j$,

\section{Network constraints at each bus}

The power balance defined by equation (5) doesn't ensure satisfying the power balance at each bus. So additional network constraints must be added at each bus $i$ as:

$$
P G_{i}=P D_{i}+\sum_{k=1}^{n c i} P F_{i k}, \text { for } \mathrm{i}=1,2 \ldots \mathrm{NB} .
$$


Where, nci represents no of lines connected with the bus $i, P D i$ is the load demand at load bus $i$ and $P F_{i k}$ is the active power flow in lines connected between buses i and $k$, respectively.

\subsection{Inequality constraints}

\section{Generation Constraints}

The generation hard constraints include generator voltages, real power outputs, these constraints are defined as hard constraints as they are restricted by their physical lower and upper limits. The generation constraints can be simulated as:

$P G_{i}^{\min } \leq P G_{i} \leq P G_{i}^{\max }$

Where, $P G_{i}{ }^{m a x}$ and $P G_{i}{ }^{m i n}$ are the maximum and minimum of power generation at bus $i$ respectively.

\section{Ramp rate Constraints}

Additional generation hard constraints are restricted by their physical ramp rate limits. The ramp rate constraints can be simulated as:

$$
D R G_{i}^{\min } \leq P G_{i}-P G_{i}^{(0)} \leq U R G_{i}^{\max }
$$

Where, $D R G_{i}^{\max }$ and $U R G_{i}^{\min }$ are the maximum and minimum of ramp rate for power generation at bus $i$ respectively. These rates are considered around $10 \%$ around the initial generation outputs $\left(P G_{\mathrm{i}}\right)$.

\section{Network Constraints}

To keep system more secure voltages at load buses and transmission line loadings should be kept in permissible limits and this can be expressed as:

$S l_{i} \leq S l_{i}^{\max }, \mathrm{i}=1,2 \ldots, \mathrm{nl}$

Where, VL is load bus voltages, $\mathrm{Sl}$ is transmission line loading in MVA, $N L$ is number of load buses, and $n_{l}$ is number of system lines.

\section{Improved Real-Coded Genetic Algorithm}

In this paper an efficient MO-RCGA operator is proposed to solve the $\mathrm{ED} / \mathrm{Emission}$ problem considering system constraints. Here, a brief summary of the genetic operators of the proposed MO-RCGA is presented. The performance of the proposed real-coded GA is an enhanced real-coded GA using biased
Initialization, adaptive parameter setting, and elitism is carried out.

\subsection{Biased initialization}

Here, it used what so called Biased Initialization. The algorithm cannot continue for evolving if the average fitness of the population does not exceed certain limit, so, at that moment you are sure somehow about the quality of the initial seed. Through different trials, it has been decided to set this threshold to be $30 \%$.

\subsection{Selection and replacement scheme}

There are two things to keep in mind during the selection of the parents, elitism and diversity. Elitism is the process of selecting the better individuals, or more to the point, selecting individual with a bias towards the better ones. Elitism is important since it allows the solutions to get better over time. If you pick only the few best parents, and replace always the worst, the population will converge quicker. This means that all the individuals will more or less all be the same. Contrarily to general belief, the solution will not necessarily be optimal. On the contrary, it can be pretty much guaranteed that it will be sub-optimal, or if you're lucky, near-optimal. This approach is fine for small problems, and small population sizes. For bigger problems, you need more diversity. This allows the genetic algorithm to search through a wider variety of solutions, and thereby not get stuck in local maxima (solutions that seem good locally, but are not in the grand scheme of things).

\subsection{Crossover}

Several genetic operators have been proposed for real-number coding, which can roughly be put into four classes [24], conventional operators, arithmetical operators, directionbased operators, and stochastic operators. Here the suggested algorithm uses Arithmetical crossover. The weighted average of two vectors $x_{1}$ and $x_{2}$ is calculated as follows:

$\lambda_{1} x_{1}+\lambda_{2} x_{2}$

If the multipliers are restricted as

$\lambda_{1}+\lambda_{2}=1, \quad \lambda_{1}>0, \quad \lambda_{2}>0$ 
Similarly, arithmetic crossover is defined as the combination of two vectors (chromosomes) as follows:

$$
\begin{aligned}
& x_{1}^{\prime}=\lambda_{1} x_{1}+\lambda_{2}^{\prime} x_{2} \\
& x_{2}^{\prime}=\lambda_{1} x_{2}+\lambda_{2}^{\prime} x_{1}
\end{aligned}
$$

\subsection{Non-uniform mutation}

Since genetic algorithms are inspired from the idea of evolution, it is natural to expect that the adaptation is used not only for finding solutions to a given problem, but also for tuning genetic algorithms to the particular problem. It is designed for fine-tuning capabilities aimed at achieving high precision. For a given parent $x$, if the element $x_{k}$ of it is selected for mutation, the resultant offspring is $x^{\prime}=\left(x_{1}, \ldots, x_{k}^{\prime}, \ldots, x_{k}\right)$, where $x_{k}^{\prime}$ is randomly selected from the following two possible choices [30, 34, 35]:

$x_{k}^{\prime}=x_{k}+\Delta\left(t, x_{k}^{U}-x_{k}\right)$ if a random bit is $0(15)$

$x_{k}^{\prime}=x_{k}-\Delta\left(t, x_{k}-x_{k}^{L}\right)$ if a random bit is $1(16)$

The function $\Delta(t, y)$ returns a value in the range $[0, y]$ such that the value of $\Delta(t, y)$ approaches 0 as $t$ increases ( $t$ is the generation number). This property causes the operator to search the space uniformly initially (when $t$ is small), and very locally at later stages. The function $\Delta(t, y)$ is given as:

$$
\Delta(t, y)=y \cdot r^{\left(1-\frac{t}{T}\right)^{b}}
$$

Where, $r$ is a random number from [0,1], $\mathrm{T}$ the maximum generation number, and $b \quad a$ parameter determining the degree of nonuniformity. It is possible for the operator to generate an offspring that is not feasible. In such a case, we can reduce the value of random number r. As binary coded GA has severe drawbacks due to the existence of Hamming Cliff, phenotype and genotype are different, precision is limited by the binary representation of variables, and the low speed comparable to Real-coded GA, the thesis uses the Real-coded GA and develops it by adding some features which help in not stuck in the local minima.

\subsection{Handling of constraints}

The proposed MO-RCGA deals efficiently with the inequality constraints. The equality constraints are treated as close to inequality constraints as given below:

$\sum P_{g}-\sum$ Load - Losses $>\epsilon$

Where, $\epsilon$ refers to the convergence degree

\subsection{Handling of conflicting objectives}

The typical optimal EED problem can be formulated as a bi-criteria optimization model. The two conflicting objectives, i.e., fuel cost and pollutants emission, should be minimized simultaneously while fulfilling certain system constraints. The EED problem has two objective functions fuel cost minimization and emission minimization. These two objectives are conflicted in nature. The mathematical formulation of multiobjective EED problem minimizes both non smooth fuel cost function (Equ. 3) and emission (Equ. 4) while satisfying system operating constraints presented in Equs. (5)-(10). The weighted-sum method transforms a set of objectives into a single objective by pre-multiplying each objective with usersupplied weight. The weight of an objective is usually chosen in proportion to the objective's relative importance to the problem. It is likely that each objective function takes different magnitude as in the combined economicenvironmental power dispatch. Therefore, setting up an appropriate weight factor depends on the scaling of each objective function. It is the usual practice to choose weights such that their sum is equal to one. The combined EED problem can be formulated as follows:

$$
F=w_{1} \times F_{1}(\text { Fuel })+w_{2} \times F_{2}(\text { Emission })
$$

Where, $\mathrm{F}$ refers to the combined objective function involves fuel cost and emission; $\mathrm{w}_{1}$ and $\mathrm{w}_{2}$ is the weighing factors of the two objective functions.

\section{Case Studies}

\subsection{Test systems}

In order to show the effectiveness of the proposed approach to solve EED problems using an MO-RCGA, Simulations were performed on the standard IEEE 30-bus 6generator test system [28]. The Six generators located at buses 25-30 as shown in the single line diagram presented in Figure 1. The power system is interconnected by 41 transmission lines and the total system demand for the 21 load buses is 283.4 MW. Fuel cost and 
emission coefficients are given in Tables 1 and 2 respectively as [20].

Table 1. Generator fuel cost coefficients

\begin{tabular}{|c|c|c|c|c|c|c|c|}
\hline Generator & $\begin{array}{c}\text { Min } \\
\text { MW }\end{array}$ & $\begin{array}{c}\text { Max } \\
\text { MW }\end{array}$ & $\begin{array}{c}\text { a } \\
\$\end{array}$ & $\begin{array}{c}\mathrm{b} \\
(\$ / M W)\end{array}$ & $\begin{array}{c}\mathrm{c} \\
\left(\$ / \mathrm{MW}^{2}\right)\end{array}$ & $\begin{array}{c}\mathrm{d} \\
\$\end{array}$ & $\begin{array}{c}\mathrm{e} \\
\mathrm{MW}^{-1}\end{array}$ \\
\hline $\mathrm{G}_{1}$ & 5 & 50 & 100 & 200 & 10 & 32.4 & 0.047 \\
\hline $\mathrm{G}_{2}$ & 5 & 60 & 120 & 150 & 10 & 32.4 & 0.047 \\
\hline $\mathrm{G}_{3}$ & 5 & 100 & 40 & 180 & 20 & 32.4 & 0.063 \\
\hline $\mathrm{G}_{4}$ & 5 & 120 & 60 & 100 & 10 & 23.4 & 0.063 \\
\hline $\mathrm{G}_{5}$ & 5 & 100 & 40 & 180 & 20 & 24 & 0.063 \\
\hline $\mathrm{G}_{6}$ & 5 & 60 & 100 & 150 & 10 & 24 & 0.063 \\
\hline
\end{tabular}

Table 2. Generator emission coefficients

\begin{tabular}{|c|c|c|c|c|c|c|}
\hline Coefficient & $\mathrm{G}_{1}$ & $\mathrm{G}_{2}$ & $\mathrm{G}_{3}$ & $\mathrm{G}_{4}$ & $\mathrm{G}_{5}$ & $\mathrm{G}_{6}$ \\
\hline$\alpha$ & 4.091 & 2.543 & 4.258 & 5.326 & 4.258 & 6.131 \\
\hline$\beta$ & -5.554 & -6.047 & -5.094 & -3.550 & -5.094 & -5.555 \\
\hline$\gamma$ & 6.490 & 5.638 & 4.586 & 3.380 & 4.586 & 5.151 \\
\hline$\xi$ & $2.0 \mathrm{E}-4$ & $5.0 \mathrm{E}-4$ & $1.0 \mathrm{E}-6$ & $2.0 \mathrm{E}-3$ & $1.0 \mathrm{E}-6$ & $1.0 \mathrm{E}-5$ \\
\hline$\lambda$ & 2.857 & 3.333 & 8.000 & 2.000 & 8.000 & 6.667 \\
\hline
\end{tabular}

\subsection{Studied cases}

To demonstrate the effectiveness of the proposed version of the MO-RCGA, Six studied cases are considered for the studied test system of the combined EED minimization problem. In Case 1, the fuel cost only is minimized for lossless network. In Case 2 while the fuel cost is minimized the transmission losses are considered. For Cases 3 and 4, minimization of emission is considered for lossless and lossy networks, respectively. In Cases 5 and 6, both objectives are optimized simultaneously with equal priority with both conditions of transmission losses, respectively.

\subsection{Setting of proposed RCGA parameters}

The proposed MO-RCGA-based approach has been developed and implemented using the MATLAB software for the studied case. Initially, several runs have been done with different values of MO-RCGA key parameters such as Chromosome numbers (candidate solution), Number of parents in selection, size of population, Elitism, selection, crossover and mutation operators.

Table 3 presents the simulation parameters of MORCGA considering lossless and lossy network.
Table 3. MO-RCGA parameters for studied cases

\begin{tabular}{|c|c|c|}
\hline Variables & Lossless Cases & Lossy Cases \\
\hline No. of runs & 50 & 50 \\
\hline Chromosome No. & 30 & 60 \\
\hline Parents no. & 26 & 56 \\
\hline population No. & 30,40 & $30-80$ \\
\hline Elitism & 4 & 4 \\
\hline Selection & Roulette-wheel & Roulette-wheel \\
\hline Crossover & arithmetic crossover & arithmetic crossover \\
\hline Mutation & Non-uniform mutation & Non-uniform mutation \\
\hline
\end{tabular}

\subsection{Results \& discussion}

Table 4 shows the EED solution solved through the proposed MO-RCGA for Case 1 at two population size (30 and 40). As seen, the proposed MO-RCGA method gets different load dispatch settings. As an operating result, the fuel cost is $582.92 \$ / \mathrm{hr}$ and the pollutant emission is $221.21 \mathrm{~kg} / \mathrm{hr}$ for Case 1 which population size equals 30 . When the population size increased to 40, the fuel cost is $585.3574 \$ / \mathrm{hr}$ at the same pollutant emission which equals $216.7 \mathrm{~kg} / \mathrm{hr}$ when the transmission losses is neglected. It is cleared that, less significant variation is found with increased population size. In terms of the control variable settings, different security levels are obtained for especially for generators 1, 2 and 3. Also, Table 4 presents the evaluation of the proposed MO-RCGA in terms of mean, best and worst values for 50 runs and the related standard deviation for each population size.

Table 4. EED solution of Case 1

\begin{tabular}{|c|c|c|}
\hline Variable & POP 30 & POP 40 \\
\hline PG $_{1}$ (per unit) & 0.1649 & 0.2334 \\
\hline $\mathrm{PG}_{2}$ & 0.2317 & 0.2336 \\
\hline $\mathrm{PG}_{3}$ & 0.4787 & 0.5749 \\
\hline $\mathrm{PG}_{4}$ & 1.0019 & 0.9400 \\
\hline $\mathrm{PG}_{5}$ & 0.4874 & 0.4221 \\
\hline $\mathrm{PG}_{6}$ & 0.371 & 0.334 \\
\hline Mean (Fuel cost) \$/hr & 593.4 & 591.3457 \\
\hline Best (Fuel cost) \$/hr & $\mathbf{5 8 2 . 9 2}$ & $\mathbf{5 8 5 . 3 5 7 4}$ \\
\hline Worst (Fuel cost) \$/hr & 602.13 & 602.7309 \\
\hline Standard-deviation & 6.5888 & 6.1059 \\
\hline Emission at best fuel costs $\mathrm{kg} / \mathrm{hr}$ & 221.2 & 216.7 \\
\hline
\end{tabular}

Considering the transmission losses, for Case 2, Table 5 presents the EED solution at three population sizes 60,70 and 80 . For all population sizes, both fuel cost and pollutant emission are increased when the transmission losses are noticed. The lowest incremental fuel costs and emissions are $615.5482 \$ / \mathrm{hr}$ and $228.5 \mathrm{~kg} / \mathrm{hr}$, respectively which are occurred at 60 populations. When the increased population size equals 70 , significant reduction in the emission level to $207.7 \mathrm{~kg} / \mathrm{hr}$. The lowest standard deviation level in Case 2 is 5.7289 which is less than the corresponding one in Case 1 (6.1059) 
Table 5. EED solution of Case 2

\begin{tabular}{|c|c|c|c|}
\hline Variables & POP 60 & POP 70 & POP 80 \\
\hline $\mathrm{PG}_{1}$ & 0.1727 & 0.2885 & 0.2311 \\
\hline $\mathrm{PG}_{2}$ & 0.3966 & 0.2688 & 0.3805 \\
\hline $\mathrm{PG}_{3}$ & 0.5679 & 0.4241 & 0.5909 \\
\hline $\mathrm{PG}_{4}$ & 1.1079 & 0.8285 & 0.9374 \\
\hline $\mathrm{PG}_{5}$ & 0.2194 & 0.6143 & 0.5939 \\
\hline $\mathrm{PG}_{6}$ & 0.3949 & 0.4351 & 0.1259 \\
\hline Mean (Fuel cost) \$/hr & 623.3722 & 625.4780 & 626.8446 \\
\hline Best (Fuel cost) & 615.5482 & 616.2909 & 618.1290 \\
\hline Worst (Fuel cost) & 634.9026 & 649.3181 & 648.0311 \\
\hline Standard-deviation & 5.7289 & 9.9135 & 9.2004 \\
\hline Emission at best Fuel costs $\mathrm{kg} / \mathrm{hr}$ & 228.5 & 207.7 & 218.7 \\
\hline
\end{tabular}

In Table 6, Cases 3 and 4 considers the emission minimization only as a single objective, the fuel costs are increased to 643.1819 and $648.5301 \$ / \mathrm{hr}$ while the pollutant emission is reduced to the lowest levels at $193.2 \mathrm{~kg} / \mathrm{hr}$ in Case 4.

Table 6. EED solution of Cases 3 and 4 at Pop.Size $=30$

\begin{tabular}{|c|c|c|}
\hline \multirow{2}{*}{ Variables } & Case 3 & Case 4 \\
\hline & POP 30 & POP 30 \\
\hline$F_{g_{k}}$ & 0.3875 & 0.3969 \\
\hline$F_{g_{x}}$ & 0.5103 & 0.4566 \\
\hline$F_{\text {gi: }}$ & 0.4776 & 0.6015 \\
\hline$F_{a_{n}}$ & 0.4773 & 0.3853 \\
\hline$F_{\text {g: }}$ & 0.51 & 0.5366 \\
\hline$F_{a i}$ & 0.5166 & 0.5064 \\
\hline Mean (Emission) & 0.2029 & 0.2018 \\
\hline Best (Emission) & 0.1934 & 0.1932 \\
\hline Worst (Emission) & 0.2152 & 0.2194 \\
\hline Standard-deviation & 0.0052 & 0.0056 \\
\hline Fuel cost (without valve effect) & 643.1819 & 648.5301 \\
\hline Fuel cost (with valve effect) & 681.7049 & 691.3766 \\
\hline
\end{tabular}

Table 7 show the compromised EED solution solved using the proposed MO-RCGA for Cases 5 and 6, respectively for varied population size 30, 40 and 50. As seen in Table 7, the proposed MORCGA method gets different load dispatch level for each population size. As an operating result, the fuel cost has the lowest level $(582.41 \$ / \mathrm{hr})$ when the population size equals 30 . The pollutant emission is $220 \mathrm{~kg} / \mathrm{hr}$ for Case 5 which population size equals 30 . When the population size equals 40 , the fuel cost is increased by 0.086 $\$ / \mathrm{hr}$ at pollutant emission is reduced by $0.8 \mathrm{~kg} / \mathrm{hr}$.

It is cleared that, less significant variation is found with increased population size. In terms of the control variable settings, different security levels are obtained for generators 1,2 and 3.

Table 7. Compromised EED solution (Cases 5 and 6)

\begin{tabular}{|c|c|c|c|c|c|c|}
\hline Variable & \multicolumn{3}{|c|}{ Case 5 } & \multicolumn{3}{c|}{ Case 6 } \\
\cline { 2 - 7 } & POP30 & POP 40 & POP50 & POP30 & POP40 & POP50 \\
\hline PG $_{\mathbf{1}}$ & 0.1461 & 0.1439 & 0.1847 & 0.1964 & 0.1468 & 0.138 \\
\hline PG $_{\mathbf{2}}$ & 0.2795 & 0.3148 & 0.3099 & 0.2512 & 0.2715 & 0.2714 \\
\hline PG $_{3}$ & 0.4714 & 0.5446 & 0.5172 & 0.4399 & 0.5046 & 0.5399 \\
\hline PG $_{4}$ & 0.9783 & 0.9776 & 0.9691 & 0.9134 & 0.9461 & 1.0308 \\
\hline PG $_{5}$ & 0.5448 & 0.3943 & 0.4782 & 0.5566 & 0.5711 & 0.4398 \\
\hline PG $_{6}$ & 0.315 & 0.3588 & 0.2753 & 0.4089 & 0.3252 & 0.347 \\
\hline (Fuel cost) & 582.41 & 582.496 & 583.17 & 591.05 & 589.31 & 589.42 \\
\hline $\begin{array}{c}\text { Emission } \\
\text { kg/hr }\end{array}$ & 220 & 219.2 & 218.5 & 214.2 & 218 & 223.4 \\
\hline
\end{tabular}

Considering the transmission losses, for Case 6, Table 8 presents the compromised EED solution. For all population sizes, both fuel costs and pollutant emission are increased when the transmission losses are considered as presented in Table 8 . The lowest fuel costs and emissions are $589.31 \$ / \mathrm{hr}$ and $218 \mathrm{~kg} / \mathrm{hr}$, respectively which are occurred at the population size equals 40 .

\section{Evaluation Criteria of MO-RCGA}

\subsection{Numerical comparative study}

The proposed MO-RCGA is compared with the results obtained with multi-objective evolutionary algorithms like non-dominated sorting genetic algorithm (NSGA) [20], niched Pareto genetic algorithm (NPGA) [20], strength Pareto evolutionary algorithm (SPEA) [20] and multiobjective fuzzy based on particle swarm optimization algorithm [10], and Modified Shuffled Frog Leaping Algorithm (MSFLA) [22].

Tables 9 shows the comparison between EED solutions solved through the proposed MORCGA against several optimization techniques for Cases 1 and 3. The use of the proposed MO-RCGA leads to more economical solutions compared to other techniques.

Table 8. Comparison of best solution for fuel cost and emission with several algorithms for Case 1 and 3 (without valve effects)

\begin{tabular}{|l|c|c|c|c|}
\hline \multirow{2}{*}{ Algorithm } & \multicolumn{2}{|c|}{ Case 1 } & \multicolumn{2}{c|}{ Case 3 } \\
\cline { 2 - 5 } & $\begin{array}{c}\text { Cost } \\
(\$ / \mathrm{h})\end{array}$ & $\begin{array}{c}\text { Emission } \\
(\mathrm{Ton} / \mathrm{h})\end{array}$ & $\begin{array}{c}\text { Cost } \\
(\$ / \mathrm{h})\end{array}$ & $\begin{array}{c}\text { Emission } \\
(\mathrm{Ton} / \mathrm{h})\end{array}$ \\
\hline NSGA[20] & 600.572 & 0.2228 & 639.209 & 0.19436 \\
\hline NPGA[20] & 600.259 & 0.2212 & 639.180 & 0.19433 \\
\hline SPEA [20] & 600.150 & 0.2215 & 638.507 & 0.19421 \\
\hline FCPSO[10] & 600.112 & 0.2222 & 638.262 & 0.1942 \\
\hline MO-RCGA & 598.7426 & 0.2212 & 643.181 & 0.1934 \\
\hline
\end{tabular}

Table 9. Comparison of different methods for Cases 2 and 4 considering transmission losses

\begin{tabular}{|l|c|c|c|c|}
\hline \multirow{2}{*}{ Algorithm } & \multicolumn{2}{|c|}{ Case 2 } & \multicolumn{2}{c|}{ Case 4 } \\
\cline { 2 - 5 } & $\begin{array}{c}\text { Cost } \\
(\$ / \mathrm{h})\end{array}$ & $\begin{array}{c}\text { Emission } \\
(\text { Ton/h) }\end{array}$ & $\begin{array}{c}\text { Cost } \\
(\$ / h)\end{array}$ & $\begin{array}{c}\text { Emission } \\
(\text { Ton/h) }\end{array}$ \\
\hline NSGA[20] & 600.3100 & 0.2238 & 633.8300 & 0.1946 \\
\hline NPGA[20] & 600.2200 & 0.2206 & 636.0400 & 0.1943 \\
\hline SPEA [20] & 600.3400 & 0.2241 & 640.4200 & 0.1942 \\
\hline MOSST[22] & 605.8900 & 0.2220 & 644.1100 & 0.1942 \\
\hline FCPSO [10] & 600.1300 & 0.2223 & 638.3577 & 0.1942 \\
\hline MSFLA[22] & 600.1114 & 0.22215 & 638.24254 & 0.1942 \\
\hline $\begin{array}{c}\text { Proposed MO- } \\
\text { RCGA }\end{array}$ & 611.6935 & 0.2285 & 648.5301 & 0.1932 \\
\hline
\end{tabular}

Table 10 shows the comparison between EED solutions solved through the proposed MORCGA against several optimization techniques for Cases 2 and 4. The use of the proposed 
MO-RCGA leads to more economical solutions compared to other techniques.

Tables10 shows the comparison between EED solutions solved through the proposed MORCGA against several optimization techniques for Cases 5 and 6. The use of the proposed MO-RCGA leads to more economical solutions compared to other techniques.

Table 10. Comparison of different methods for the best compromise solution

\begin{tabular}{|l|c|c|}
\hline Algorithm & Emission (Ton/h) & Cost (\$/h) \\
\hline SPEA [20] & 0.2004 & 610.3 \\
\hline NSGA [20] & 0.2041 & 606.03 \\
\hline NPGA [20] & 0.2015 & 608.90 \\
\hline MSFLA [22] & 0.2006 & 610.0783 \\
\hline MO-RCGA for Case 5 & 0.2159 & 578.8774 \\
\hline MO-RCGA for Case 6 & 0.2186 & 587.0558 \\
\hline
\end{tabular}

\subsection{Convergence criteria for MO-RCGA}

Figures 2-9 show the convergence of objective function/s for the studied cases. Figure 2 shows the convergence at the best fuel cost for Case 1 at population number 20 and 30 when the transmission losses is not considered where the population size equals 30. Figure 3 shows the convergence at the best fuel cost for Case 1 at population number 30 and 40 when the transmission losses is considered where the population size equals 40 . Figure 4 shows the convergence at the best fuel cost for Case 2 at population number 50 and 60 when the transmission losses is considered where the population size equals 60. Figure 5 shows convergence of best emission for Case 3 at population sizes 30 and 40 . The best emission for 50 runs is presented in Figure 6. Figure 7 shows the convergence best emission points at population size 20 and 30 for Case 4 .

Figures 8 and 9 show the fuel costs against emission for cases 5 and 6 at different preference value, respectively.

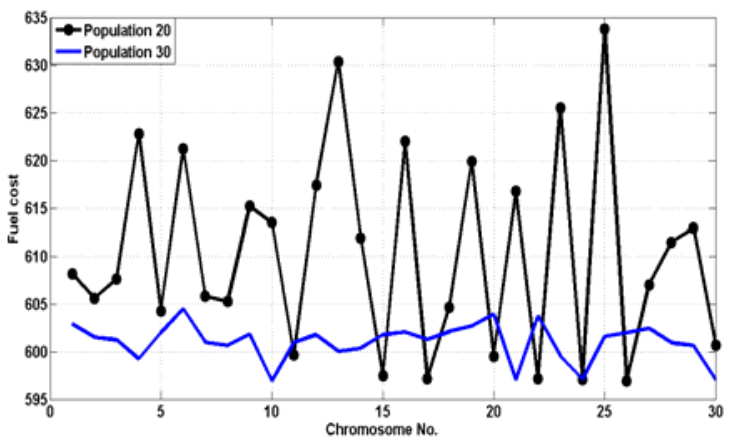

Figure 2. Convergences for Case 1 at population no 20 and 30.

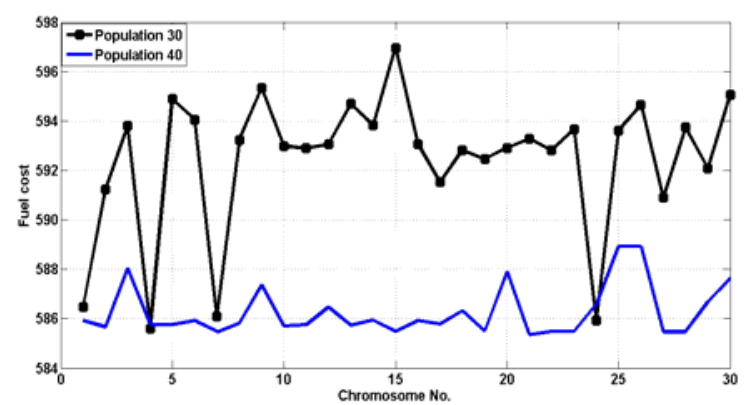

Figure 3. Convergence for Case 1 at population no 30 and 40

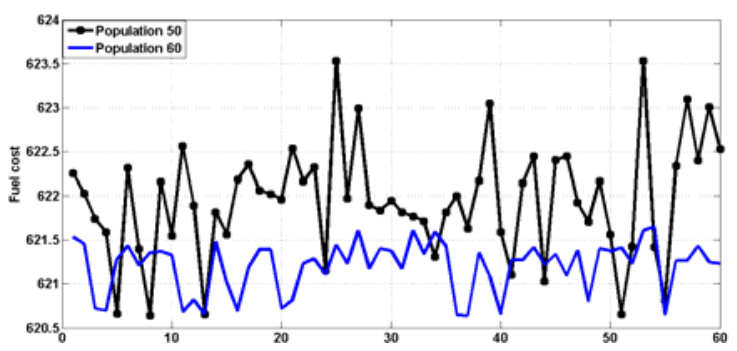

Figure 4. Convergence for Case 2 at population no 50 and 60

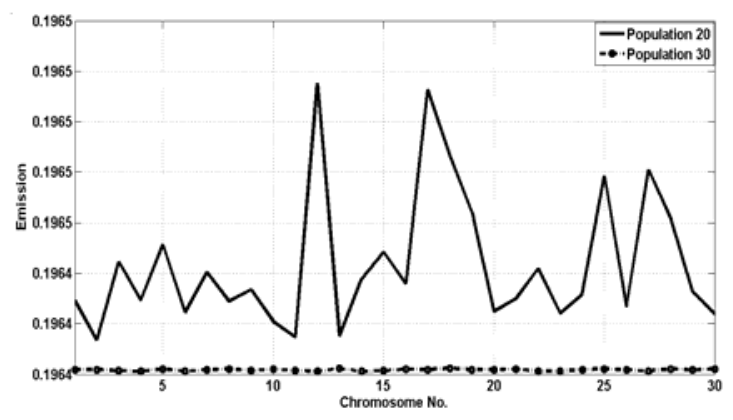

Figure 5. Convergence for Case 3 at population no 30 and 40

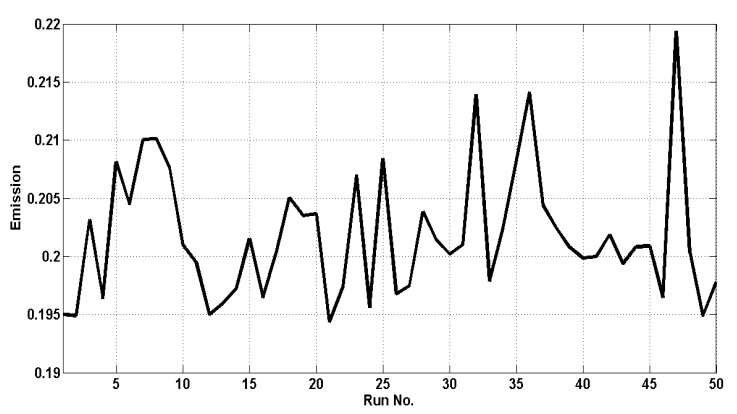

Figure 6. Best emission levels for 50 runs (Case 4)

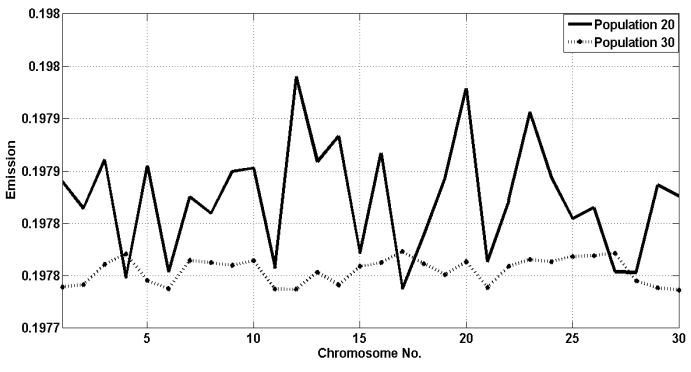

Figure 7. Convergence best emission points at population size 20 and 30 for Case 4 


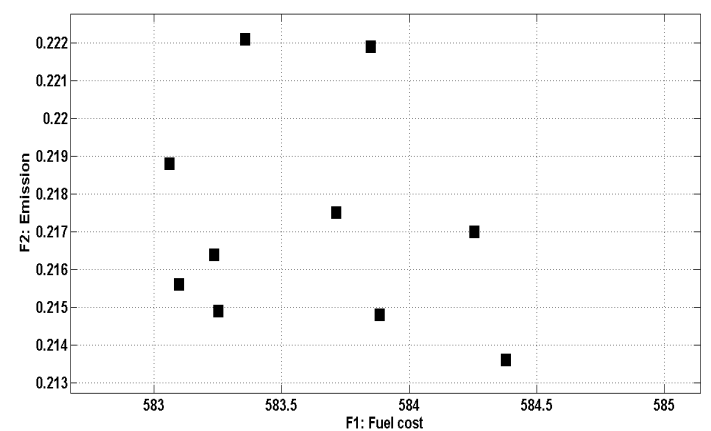

Figure 8. Emission versus fuel costs for Case 5.

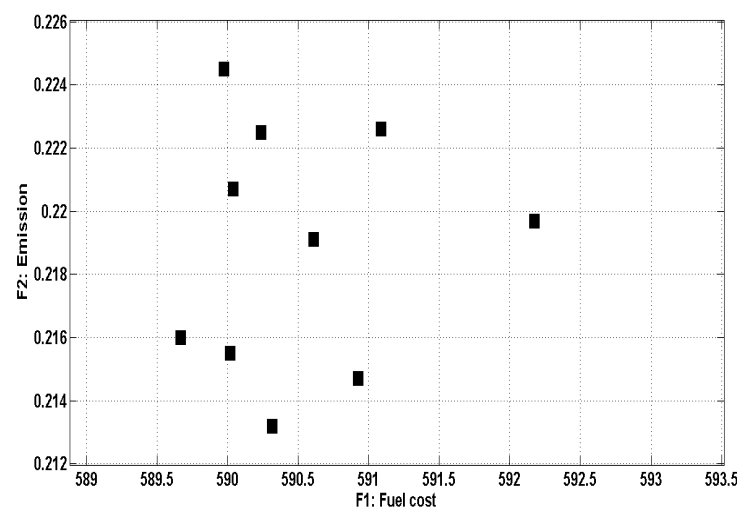

Figure 9. Emission versus fuel costs for Case 6

\section{Conclusion}

In this paper, an improved multi-objective real coded genetic algorithm has been presented and efficiently examined for the combined competing economic/environmental objectives in power dispatch problem. The multi-objective optimization problem was transformed in to single objective using the weighted-sum method. The proposed RCGA has been developed, and successfully tested on the standard IEEE 30-bus test system. Several studied cases have been considered, the simulation results demonstrate the effectiveness and robustness of the proposed MO-RCGA algorithm.

\section{REFERENCES}

1. WOOD, A. J., B. F. WOLLENBERGY, Power Generation, Operation, and Control. New York: Wiley, 1984.

2. BASU, M., Economic Environmental Dispatch using Multi-objective Differential Evolution, Applied Soft Computing, vol. 11, 2011, pp. 2845-2853.

3. ZHU, J., Optimization of Power System Operation, IEEE Press Series on Power Engineering, 2009.
4. ABIDO, M. A., A Niched Pareto Genetic Algorithm for Multiobjective Environmental/Economic Dispatch, Electrical Power and Energy Systems, vol. 25, 2009, pp. 97-105.

5. GJORGIEV, B., M. CEPIN, A Multiobjective Optimization based Solution for the Combined EconomicEnvironmental Power Dispatch Problem, Engineering Applications of Artificial Intelligence, vol. 26, 2013, pp. 417-429.

6. WU, L. H., Y. N. WANG, X. F. YUAN, S. W. ZHOU, Environmental/Economic Power Dispatch Problem using Multiobjective Differential Evolution Algorithm, Electric Power Systems Research, vol. 80, 2010, pp. 1171-1181.

7. YANJUN, J., J. JIANG, Y. ZHANG, A Novel Fuzzy Multiobjective Model using Adaptive Genetic Algorithm Based on Cloud Theory for Service Restoration of Shipboard Power Systems, IEEE Translations on Power Systems, vol. 20, 2012, pp. 612-620.

8. AMJADY, N., H. NASIRI-RED, Nonconvex Economic Dispatch with AC Constraints by a New Real Coded Genetic Algorithm, IEEE Trans. on Power Systems, vol. 24, 2009, pp. 1489-1502.

9. ABOU EL-ELA, A. A., M. BISHR, S. ALLAM, R. EL-SEHIEMY, Optimal Preventive Control Actions in Power System using Multi-Objective Fuzzy Linear Programming Technique, Electric Power Systems Research, vol. 74, 2005, pp. 147-155.

10. AGRAWAL, S., B. K. PANIGRAHI, M. K. TIWARI, Multiobjective Particle Swarm Algorithm with Fuzzy Clustering for Electrical Power Dispatch, IEEE Trans. on Evolutionary Computation, vol. 12, 2008, pp. 529-541.

11. CHATURVEDI, K. T., M. PANDIT, L. SRIVASTAVA, Self-Organization

Hierarchical Particle Swarm Optimization for Nonconvex Economic Dispatch, IEEE Trans. on Power Systems, vol. 23, 2008, pp. 1079-1087.

12. MENG, K., H. G. WANG, Z. Y. DONG, K P. WONG, Quantum-Inspired Particle Swarm Optimization for Valve-Point 
Economic Load Dispatch, IEEE Trans. on Power Systems, vol. 20, 2010, pp. 215-222.

13. VLACHOGIANNIS, J. G., K. Y. LEE, Economic Load Dispatch-A Comparative Study on Heuristic Optimization Techniques With an Improved Coordinated AggregationBased PSO, IEEE Trans. on Power Systems, vol. 24, 2009, pp. 991-1001.

14. BHATTACHARYA, A., P. KUMAR CHATTOPADHYAY, Biogeographybased Optimization for Different Economic Load Dispatch Problems, IEEE Trans. on power systems, vol. 25, 2010, pp. 1064-1077.

15. BHATTACHARYA, A., P. KUMAR CHATTOPADHYAY, Hybrid Differential Evolution with Biogeography-based Optimization for Solution of Economic Load Dispatch, IEEE Trans. on Power Systems, vol. 25, 2010, pp. 1955-1964.

16. DUVVURU, N., K. S. SWARUP, A Hybrid Interior Point Assisted Differential Evolution Algorithm for Economic Dispatch, IEEE Trans. Power systems, vol. 26, 2011, pp. 542-549.

17. POTHIYA, S., I. NGAMROO, W. KONGPRAWECHNON, Ant Colony Optimization for Economic Dispatch Problem with Non-smooth Cost Functions, International Journal of Electrical Power and Energy System, vol. 32, 2010, pp. 478-487.

18. SHAW, B., V. MUKHERJEE, S. P. GHOSHAL, Solution of Economic Dispatch Problems by Seeker Optimization Algorithm, Expert Systems with Applications, vol. 39, 2012, pp. 508-519.

19. YANG, X., S. S. S. HOSSEINI, A. H. GANDOMI, Firefly Algorithm for Solving Non-convex Economic Dispatch Problems with Valve Loading Effect, Applied Soft Computing, vol. 12, 2012, pp. 180-1186.
20. ABIDO, M. A., Multiobjective Evolutionary Algorithms for Electric Power Dispatch Problem. IEEE Trans. on Evolutionary Computation, vol. 10, 2006, pp. 315-329.

21. HOTA, P. K., A. K. BARISAL, R. CHAKRABARTI, Economic Emission Load Dispatch through based Bacterial Foraging Algorithm, International Journal of Electrical Power \& Energy Systems, vol. 32, 2010, pp. 794-803.

22. SRINIVASA REDDY, A., K. VAISAKH, Economic Emission Load Dispatch by Modified Shuffled Frog Leaping Algorithm, International Journal of Computer Applications, vol. 31, 2011, pp. 58-65.

23. CHONG, E. K. P., S. H. ZAK, An Introduction to Optimization, $2^{\text {nd }}$ Ed., Wiley Interscience Series In Discrete Mathematic and Optimization, 2001.

24. HAUPT, R. L., S. E. HAUPT, Practical Genetic Algorithms, $2^{\text {nd }}$ Edition, Wiley Interscience, 2004.

25. EIBEN, A. E., R. HINTERDING, Z. MICHALEWICZ, Parameter Control in Evolutionary Algorithms, IEEE Trans. on Evolutionary Computation, vol. 3(2), July 1999, pp. 124-141.

26. GEN, M., R. CHENG, Genetic Algorithms and Engineering Optimization, A Wiley-Interscience Publication, 2000.

27. DREOET, J. Al., Metaheuristics for Hard Optimization, Springer-Verlag Berlin Heidelberg, 2006.

28. Washington University Website: www.ee.washington.edu/research/pstca/ 